\title{
SOZIALE KONTAKTE ALS WEG AUS DER ERWERBSLOSIGKEIT*
}

\author{
Martina Brandt
}

\begin{abstract}
Zusammenfassung: Gerade Erwerbslose werden selten berücksichtigt, wenn es um die Untersuchung des Nutzens sozialer Kontakte für die Verbesserung der Arbeitsmarktposition geht. In diesem Artikel wird anhand der Längsschnittdaten des deutschen Niedrigeinkommens-Panels 19982002 gezeigt, dass sich Granovetters These der „Stärke schwacher Beziehungen“ (1973) modifizieren und auf Erwerbslose mit niedrigem Einkommen übertragen lässt. Etwa ein Drittel der arbeitslosen Befragten findet einen neuen Job mithilfe sozialer Kontakte. Ereignisanalysen zeigen, dass der Ausstieg aus Erwerbslosigkeit in der Beobachtungszeit umso eher stattfindet, a) je mehr enge Kontakte ein Erwerbsloser besitzt und b) je breiter seine Beziehungen gestreut sind. Der signifikante positive Effekt der sozialen Netzwerke bleibt unter Kontrolle von wichtigen Prädiktoren für den Arbeitsmarkterfolg wie Dauer der Arbeitslosigkeit, Gesundheitszustand, Bildung, Geschlecht, Alter und Aktivitäten der öffentlichen Arbeitsvermittlung stabil.
\end{abstract}

\section{Einleitung: Arbeitslosigkeit und soziale Beziehungen}

Arbeitslosigkeit kann jeden treffen. Die Protestwellen gegen die Hartz IV-Reform zeigten deutlich, von welcher Brisanz das Thema Arbeitslosigkeit und der politische Umgang mit ihr für die gesamte deutsche Bevölkerung heute ist (z.B. Niejahr und Rudzio 2005).

In der BRD hat mit steigenden Arbeitslosenzahlen, sinkendem Erfolg der öffentlichen Arbeitsvermittlung und verstärkt durch den Vermittlungsskandal des Arbeitsamtes im Februar 2002 die Diskussion um mögliche Maßnahmen zur effizienteren Wiedereingliederung Arbeitsloser in den Arbeitsmarkt immer mehr Aufmerksamkeit auf sich gezogen. Hierbei treten kommunale Vermittlungsstrukturen, regionale Netzwerke und die Förderung individueller Suchaktivitäten weiter in den Vordergrund des Interesses (z.B. Niejahr 2003). Die Nutzung sozialer Kontakte kann dem Einzelnen entscheidende Vorteile im Wettbewerb um knappe Güter, wie z.B. Arbeitsplätze, bringen (Deeke

* Dieser Artikel ist im Rahmen des Projektes „Ausstiege aus dem unteren Einkommensbereich: Individuelle Aktivitäten, soziale Netzwerke und institutionelle Hilfeangebote" unter Leitung von Prof. Dr. Hans-Jürgen Andreß (Lehrstuhl für Empirische Sozial- und Wirtschaftsforschung, Universität zu Köln) entstanden. Das Projekt wurde aus Mitteln der Hans-BöcklerStiftung gefördert. Ich möchte mich bei Hans-Jürgen Andreß, Christian Deindl, Klaus Haberkern, Anne Krüger, Silke Schneider und Marc Szydlik für ihre Unterstützung bedanken. Dank gilt weiterhin den Gutachtern und Herausgebern, die wertvolle Anregungen zur Verbesserung des Artikels gegeben haben.

Kölner Zeitschrift für Soziologie und Sozialpsychologie, Jg. 58, Heft 3, 2006, S. 468-488. 
1991: 9). Die Stärke der Auswirkungen sozialer Beziehungen im Vergleich zu Persönlichkeitsmerkmalen und anderen Randbedingungen ist jedoch umstritten. Auch ist unklar, welche Merkmale ein soziales Netzwerk aufweisen muss, um seine positive Wirkung zu entfalten.

Um zu untersuchen, inwiefern soziale Kontakte ein wirkungsvolles Hilfsmittel für den Ausstieg aus Erwerbslosigkeit sein können, werden hier die Daten des Niedrigeinkommens-Panels (NIEP) benutzt. Es handelt sich dabei um Individual- und Haushaltsdaten, die von Infratest Sozialforschung im Auftrag des Bundesministeriums für Arbeit und Sozialordnung erhoben wurden und das untere Einkommensquintil in Deutschland von 1998 bis 2002 abbilden. Ziel war es, eine tragfähige Datengrundlage zu schaffen, um effektive „Maßnahmen zur Bekämpfung von Armut, Sozialhilfebedürftigkeit und Niedrigeinkommen“ (Kortmann et al. 2002: 7) erarbeiten zu können.

Wenn es um die Nutzung von sozialen Kontakten auf dem Arbeitsmarkt geht, konzentriert sich die empirische Forschung zumeist auf hoch gebildete und/oder ökonomisch gut gestellte Personen und deren Jobeinstieg oder -wechsel (z.B. Granovetter 1974; De Graaf und Flap 1988; ein Überblick in Haug und Kropp 2002). Aber „der Kontakt über Bekannte etc. zum Betrieb, in dem der neue Arbeitsplatz gefunden wurde, ist nicht die Domäne der Personen mit höheren Bildungsabschlüssen oder mit anspruchsvolleren Berufsausbildungen“ (Blaschke 1987: 167, Hervorhebung im Original).

Diese Arbeit ist eben dieser wenig beachteten Thematik gewidmet: Erstens wird die Existenz sozialer Kontakte bei Niedrigeinkommensbeziehern und zweitens ihr Einfluss auf den Erfolg der Stellensuche untersucht. Die zentrale Frage ist also, aufgrund welcher Eigenschaften und inwieweit soziale Beziehungen dem Einzelnen zum Ausstieg aus Erwerbslosigkeit und somit auch zum Ausstieg aus einer prekären Einkommenslage verhelfen können.

Ausgehend von der These der Stärke schwacher Beziehungen (Granovetter 1973) und deren Erweiterung (z.B. Wegener 1987, 1989, 1991) werden im ersten Teil des Artikels die theoretischen Erwartungen für die Untersuchungsgruppe formuliert, die dann im zweiten Teil empirisch überprüft werden.

\section{Soziale Beziehungen und Arbeitsmarkt}

\section{Soziale Beziehungen als Ressource}

Das Konzept der „embeddedness“ (Granovetter 1992) macht den Zusammenhang zwischen sozialen Beziehungen und Prozessen auf dem Arbeitsmarkt unmittelbar deutlich: „Economic action is socially situated and cannot be explained by reference to individual motives alone. It is embedded in ongoing networks of personal relationships rather than being carried out by atomized actors" (Granovetter und Swedberg 1992: 9).

So können soziale Netzwerke auch die Arbeitsmarktposition des Einzelnen beeinflussen: Nach Lin (2001: 20) sind die drei wichtigsten Eigenschaften sozialer Beziehungen für die Stellensuche: a) die Verbesserung des Informationsflusses, b) die Einflussnahme von sozialen Kontakten auf Entscheidungsträger („ein gutes Wort einlegen“) 
und c) die symbolhafte Bestätigung der sozialen Kompetenzen bzw. der Hinweis auf Möglichkeiten des Stellenanwärters, auf soziale Ressourcen zurückgreifen zu können, die für den Arbeitgeber nützlich sein könnten („soziales Zeugnis“). Kontakte können also als Ressource verstanden werden, die für den Ausstieg aus Erwerbslosigkeit von Bedeutung ist.

2. Die Stärke schwacher Beziehungen und Weiterentwicklungen

Nach „The Strength of Weak Ties“ (Granovetter 1973, 1974, 1983) haben bei der Stellensuche vor allem die Beziehungen, die von geringer Kontakthäufigkeit und schwacher emotionaler Bindung gekennzeichnet sind (Granovetter 1973: 1361), eine Bedeutung auf dem Arbeitsmarkt. Nur diese verhelfen dem Stellensuchenden zu neuen Informationen über freie Stellen, da sich schwach verbundene Kontaktpersonen potenziell in anderen sozialen oder lokalen Kreisen bewegen und wiederum Kontakt pflegen, auf die der Suchende sonst keinen Zugriff hätte. Sie dienen als lokale Brücken zur schnellen und effizienten Weitergabe von Informationen über Netzwerkgrenzen hinweg, da sie auf kurzem Wege Menschen großer sozialer Distanz erreichen können (Granovetter 1973: 1365f.).

Zusammengefasst formuliert Granovetter folgende Thesen (vgl. Preisendörfer und Voss 1988: 107f.):

- Über Kontakte findet sich schneller und mit geringerem Aufwand ein Arbeitsplatz.

- Über Kontakte wird eine bessere berufliche Platzierung erreicht als über andere Vermittlungswege. Dies geschieht vor allem mithilfe schwacher Beziehungen.

- Je weiter die Karriere fortgeschritten ist, desto eher findet sich eine Stelle über Kontakte.

- Zu Beginn der Karriere sind starke Beziehungen hilfreicher, während bei fortschreitender Karriere schwache Beziehungen an deren Stelle treten.

- Schwache Beziehungen spielen vor allem bei qualifizierten Berufsgruppen eine Rolle. Geht es jedoch um den Ausstieg aus Arbeitslosigkeit in einer Untersuchungsgruppe, die sich hauptsächlich aus dem „unteren“ sozialen Bereich rekrutiert, ist im Umkehrschluss anzunehmen, dass starke Beziehungen möglicherweise nutzbringender sind als schwache.

Zum einen ist denkbar, dass vor allem das relativ häufigere Vorkommen schwacher Beziehungen zwischen verschiedenen Subsystemen deren Stärke ist: „The strength of weak ties lies not in their individual efficiency but in their numbers" (Friedkin 1982: 285). Die Stärke schwacher Beziehungen resultiert dann vor allem daraus, dass sie den Netzwerkumfang stark erhöhen und somit mehr Informationsmöglichkeiten, jedoch nicht -sicherheiten schaffen. Inhaltlich jedoch leisten starke Beziehungen möglicherweise eine effizientere Weitergabe von Informationen (vgl. Liu und Duff 1972: 362) und können gar Brückenfunktion haben (z.B. Bian 1997: 367).

Zum anderen könnte eine Schichtabhängigkeit der Wirkung sozialer Beziehungen bei der Stellensuche bestehen (Wegener 1987: 282f.): Der Erfolg einer Handlung hängt vor allem von den Ressourcen der genutzten Kontakte ab („Soziale-RessourcenPrinzip“). Der Zugang zu sozialen Ressourcen ist umso besser, je höher die eigene so- 
ziale Position ist („Positionsstärke-Prinzip“). Drittens gilt das „Homophilieprinzip“: je ähnlicher die Kontaktpartner, desto stärker ist die Beziehung. Allerdings sind die $\mathrm{Zu}$ gangschancen zu weiteren Ressourcen aufgrund der Ähnlichkeit der Beziehungspartner bei starker Bindung gering. D.h. schwache Beziehungen können eher Zugang zu höheren sozialen Kreisen verschaffen.

Für die unteren sozialen Lagen folgt, dass nach dem Positionsstärke-Prinzip die Möglichkeiten eines Aufstiegs mithilfe schwacher sozialer Beziehungen stark beschränkt sind, zusätzlich schwindet gerade mit der Arbeitslosigkeit das soziale Kapital aus arbeitsbezogenen Kontakten (Gröhnke et al. 1996: 9). Zudem ist der Einzelne auf die Unterstützung durch starke Beziehungen besonders angewiesen, wenn er nur über geringe soziale Ressourcen verfügt (Wegener 1987: 289) - diese Beziehungen verlangen jedoch ein relativ hohes Maß an Zeit und Engagement (Wegener 1987: 281). Die Theorie bleibt also unvollständig, „solange sie keine Aussagen über die über starke Netzwerkbeziehungen ,erreichbaren Netzwerkpositionen' und deren Heterogenität macht" (Wegener 1989: 295).

Zusammengefasst: Wichtig ist die Menge der aus Netzwerkkontakten generierbaren Ressourcen (quantitativ), die Verschiedenartigkeit oder Heterogenität der Netzwerkbeziehungen (qualitativ) und die Schicht- und Situationsabhängigkeit (z.B. beruflicher Aufstieg, Ausstieg aus Erwerbslosigkeit etc.) der Bedeutung unterschiedlicher sozialer Beziehungen für die Stellensuche. Starke und schwache soziale Beziehungen können dem Stellensuchenden zum Informationsvorsprung und zu einstellungsrelevanten Vorteilen im Wettbewerb um knappe Arbeitsplätze verhelfen. ${ }^{1}$

\section{Forschungsstand: Soziale Kontakte und Ausstieg aus Arbeitslosigkeit}

Die bisherige empirische Forschung im Hinblick auf die Vermittlung Arbeitsloser in Deutschland findet eine Vermittlungsquote von ca. einem Drittel über soziale Kontakte (z.B. Noll 1985: 292; Blaschke 1987: 165). Verwandte, Bekannte und Freunde werden als wichtige Informationsquellen genutzt. Nach Habich (1987: 169) verwendet knapp die Hälfte der Untersuchten beim Arbeitgeberwechsel, beim Jobeinstieg und bei der Wiederaufnahme einer Erwerbstätigkeit informelle Informationen. Zudem sinkt die Dauer bis zum Wiedereintritt in das Arbeitsleben mit der Größe des sozialen Netzwerks (Tazelaar 1990: 209). Gerade die Dauer der Arbeitslosigkeit wird als besonders wichtiger Faktor für die (eingeschränkte) Möglichkeit des Wiedereinstiegs in die Erwerbstätigkeit genannt (z.B. Vishwanath 1989: 488). Dennoch wird theoretisch und empirisch gezeigt, dass soziale Kontakte auch für (Langzeit-)Arbeitslose bei der Stellensuche hilfreich sind: Arbeitslose bedienen sich sogar erfolgreich der Kontakte zu früheren Kollegen (Gröhnke und Strasser 1997: 19ff.; Runia 2002a: 93).

1 Die Problematik der Abhängigkeit sozialer Beziehungen von der ökonomischen und sozialen Position als kausal umgekehrte Betrachtungsweise (z.B. Andreß et al. 1995) wird weitgehend ausgeblendet. Dies wird ermöglicht, da sich die befragten Niedrigeinkommensbezieher vermutlich in einer untereinander vergleichbaren Lage befinden, sie haben also ähnliche (ökonomische) Ausgangsvoraussetzungen, jedoch, wie zu zeigen sein wird, unterschiedliche soziale Netzwerke und Ausstiegschancen. 
In der folgenden Analyse auf Basis des Niedrigeinkommens-Panels 1998-2002 könnte sich der Einfluss sozialer Beziehungen aufgrund gestiegener Arbeitslosigkeit nach der "These sozialer Schließung“ (Windolf und Hohn 1984, 1985) noch vergrößert haben. Hohe Arbeitslosenquoten führen hiernach zur verstärkten Nutzung "geschlossener" Rekrutierungswege, soziale Netzwerke oder betriebsinterne Ausschreibungen gewinnen dann an Bedeutung (Windolf und Hohn 1985: 309ff.). ${ }^{2}$

\section{Hypothesen zum Effekt sozialer Beziehungen bei der Stellensuche}

Wenn soziale Beziehungen ein Erfolg versprechender Weg zum Ausstieg aus Erwerbslosigkeit sind, besteht auch ein Zusammenhang zwischen unterschiedlichen Netzwerkeigenschaften wie dem Umfang oder der Verschiedenartigkeit der im Netzwerk befindlichen Beziehungen und der Ausstiegschance. ${ }^{3}$

Es muss davon ausgegangen werden, dass hier unterschiedlichste Rahmenbedingungen beeinflussend wirken: Strukturelle und persönliche Eigenschaften, wie zum Beispiel Ressourcen aller Beteiligten oder Positionierung im Netzwerk, ebenso aber auch soziale und ökonomische Ausgangslage sind Einflussfaktoren, die in der vorangegangenen theoretischen Betrachtung und in empirischen Untersuchungen identifiziert wurden.

Die übergreifende Ausgangshypothese für die folgende empirische Untersuchung lässt sich demnach wie folgt formulieren:

H1 Die Nutzung sozialer Kontakte verbessert die Erfolgsaussichten von Niedrigeinkommensbeziehern bei der Stellensuche.

Im Hinblick auf die sozialen Beziehungen werden die folgenden Hypothesen zugrunde gelegt:

$\mathrm{H} 2$ Je größer das Netzwerk des Einzelnen, desto höher ist die Wahrscheinlichkeit eines Ausstiegs aus Erwerbslosigkeit.

Der Einzelne verfügt über umso mehr potenzielle soziale Ressourcen im Hinblick auf Informationen, je mehr Kontaktpartner zu Rate gezogen werden können. Außerdem stellt die Menge der Kontaktpartner ein soziales Zeugnis und symbolhafte Bestätigung der „soft skills“ bei der Stellensuche dar.

Die Größe des Netzwerkes allein garantiert jedoch noch nicht, dass einer der möglichen Kontaktpartner auch Informationen besitzt, die für den Suchenden neu und

2 Diese These wird durchaus kritisch betrachtet und die empirischen Ergebnisse sind nicht eindeutig (z.B. Preisendörfer und Voss 1988: 111, 116). Es könnte ebenso zutreffen, dass gerade bei hohem Arbeitsangebot keine Schließung stattfindet, sondern möglichst weiträumig gesucht wird, um die bestmögliche Stellenbesetzung zu gewährleisten.

3 Der nahe liegende Einwand, dass womöglich kontaktfreudigere Menschen allein aufgrund dieser Persönlichkeitseigenschaft bessere Einstellungschancen haben, und nicht die sozialen Kontakte an sich Informationsvorteile etc. bieten, kann hier nur durch die Kontrolle von beobachteten Persönlichkeitseigenschaften in der multivariaten Analyse gemindert werden. In der Theorie wird dieses Problem eingebunden, indem vermutet wird, dass dem Arbeitgeber die „soft skills“ des Einzelnen durch viele soziale Kontakte symbolhaft bestätigt werden (vgl. Lin 2001). 
hilfreich bei der Jobsuche sind. Bewegen sich alle Netzwerkpartner in einem eingeschränkten sozialen und räumlichen Umfeld, sinkt auch bei einem großen Netzwerk die Wahrscheinlichkeit, dass Kontakte zum zukünftigen Arbeitgeber oder Informationen über eine freie Arbeitsstelle bestehen. Daraus ergibt sich:

\section{H3 Je heterogener das Netzwerk des Einzelnen, desto höher ist die Wahrscheinlichkeit ei-} nes Ausstiegs aus Erwerbslosigkeit.

Für die folgende Analyse der Stellensuche bei Niedrigeinkommensbeziehern wird also davon ausgegangen, dass starke und schwache Beziehungen hilfreich sind, da gerade in der betrachteten Gruppe starke Beziehungen aufgrund ihrer größeren Häufigkeit und ihres Verpflichtungscharakters eine wichtige Stellung einnehmen. Dies gilt allerdings unter der Einschränkung, dass ab einer gewissen Zahl die Restriktivität starker Beziehungen eine Belastung für das Individuum darstellen könnte.

\section{Daten, Operationalisierungen und Methoden}

\section{Das Niedrigeinkommens-Panel}

Im Erhebungszeitraum von November 1998 bis Juni 2002 wurden Angehörige des unteren Einkommensquintils und potenziell vom Abstieg in die „unteren 20 Prozent“ gefährdete Personen über sechs halbjährliche Wellen mithilfe computergestützter Telefoninterviews befragt. Die Grundgesamtheit sind also alle zum ersten Befragungszeitpunkt im Niedrigeinkommensbereich befindlichen Haushalte und solche, die laufende Hilfe zum Lebensunterhalt bezogen oder in denen die Bezugsperson Arbeitslosenunterstützung erhielt. ${ }^{4}$

Der jeweilige volljährige Haushaltsvorstand oder dessen Lebenspartner gaben am Telefon Auskunft über alle anderen Haushaltsmitglieder. Da wichtige Informationen nur für die befragte Bezugsperson erhoben wurden, werden in der empirischen Analyse im Anschluss nur Eigenauskünfte aufgenommen, was gerade bei Fragen zu sozialen Kontakten zu höherer Verlässlichkeit führt. Aus denselben Gründen werden nur Haushalte berücksichtigt, in denen die Befragungsperson nicht mehr als einmal gewechselt hat $(\mathrm{n}=1913)$.

Aufgrund der Auswahlkriterien (Einkommensarmut/Unterstützungsleistungen, Volljährigkeit und Deutschsprachigkeit des Haushaltsvorstandes, Festnetz-Telefonanschluss) ergibt sich eine besondere demographische Zusammensetzung der Stichprobe. Ein-Personen-Haushalte, Haushalte mit fünf und mehr Personen sowie Alleinerziehende sind im NIEP überdurchschnittlich häufig vertreten (Kortmann und Sopp 2000: 28f.), was auf vergleichsweise hohe Armutsrisiken dieser Haushalte hindeutet (vgl. auch Kaufmann 1995: 138ff.). Diese Umstände treffen Frauen in erhöhtem Maße: Nach eigenen Berechnungen sind fast 70 Prozent der Bezugspersonen in der ersten Welle weiblichen Geschlechts. Aufgrund des relativen Kinderreichtums in den Haushalten gestaltet sich

4 Weiterführende technische Informationen können im Methodenbericht (Kortmann et al. 2002) nachgelesen werden. 
auch die Altersstruktur anders als in der Gesamtbevölkerung: 45 Prozent der NIEPPopulation sind zwischen 25 und 45 Jahre alt, in der gesamtdeutschen Bevölkerung sind dies nur 35 Prozent (Kortmann und Sopp 2000: 32). Überraschenderweise entspricht das Bildungsniveau dem gesamtdeutschen Niveau fast, es befinden sich jedoch erwartungsgemäß in der Stichprobe überdurchschnittlich viele Arbeits- und Erwerbslose (30 Prozent nach Selbsteinstufung).

Im NIEP werden Informationen zum Ausstieg aus Erwerbslosigkeit über die Zeit und die Ausprägung sozialer Kontakte im Niedrigeinkommensbereich erhoben, was diese Datenquelle zu einer geeigneten (und in dieser Form einzigen) Grundlage für die hier erarbeitete Fragestellung macht. ${ }^{5}$

\section{Operationalisierungen}

a) Soziale Beziehungen. Soziale Netzwerke werden hier aus individueller Sicht erfasst: Das „egozentrierte Netzwerk“ ist „das Geflecht informeller Beziehungen des einzelnen Menschen mit Personen aus seiner sozialen Umgebung" (Diewald 1991: 59). In der dritten Welle des NIEP wird das Vorhandensein verschiedener Beziehungsarten im Ego-Netzwerk anhand von Globalfragen erhoben, die Konstanz der Netzwerke von diesem Befragungszeitpunkt an muss also für die weiteren Analysen vorausgesetzt werden.

Die Netzwerkangaben werden aus Welle 3 in die Folgewellen übertragen und alle Auswertungen beziehen sich auf Welle 4 bis Welle 6 . So ist sichergestellt, dass die in den Hypothesen vermutete Kausalrichtung, nämlich die Auswirkung sozialer Netzwerke auf den Ausstieg, überprüft wird und nicht umgekehrt. Zur Messung der Netzwerke im vorliegenden Fall werden die Größe des Netzwerks und die Heterogenität der Rollenbeziehungen verwendet (vgl. z.B. Burt 1983; Diewald 1991; Wegener 1989, 1991; Jansen 2003). ${ }^{6}$ In Tabelle 1 wird ein Überblick über die im NIEP in Welle 3 abgefragten Beziehungsarten bzw. ihre Operationalisierung für diese Analyse gegeben. Sie gehen in die Indizes für Netzwerkgröße und -heterogenität ein:

Der Größenindex bildet die Zahl der engen Kontakte ab, enge Freunde und die erwachsenen Haushaltsmitglieder gehen hier ein. Insgesamt rangiert der Index zwischen null und acht und ist ein Indiz für die starken Beziehungen im Netzwerk.7

5 Andere deutsche Erhebungen, die soziale Netzwerke in geeigneter Form abbilden (z.B. Eurobarometer 1996, Allbus), berücksichtigen den unteren Einkommensbereich nicht ausreichend, da gerade hier häufig eine besonders schlechte Ausschöpfung erreicht wird.

6 Im NIEP wurden detailliertere Eigenschaften von Beziehungen (emotionale Bindung, Kontakthäufigkeit etc.) nicht direkt erfasst, daher kann „Stärke“ vs. „Schwäche“ nur über Rollenbezeichnungen erschlossen werden. Auch die Zahl der potentiell eher schwachen Beziehungen wurde nicht erfragt.

7 Die Anzahl der engen Freunde erweist sich in der Überprüfung des Antwortverhaltens bedauerlicherweise als relativ inkonsistent und es zeigt sich, dass das Verständnis des Freundschaftsbegriffs bei verschiedenen Befragten stark differiert. Zusätzlich zu einer Tendenz zur „Verallgemeinerung", d.h. der häufigen Nennung von zehn und zwanzig Freunden trotz insgesamt seltener Nennungen (10 Prozent > 5, 2 Prozent $>10)$ der oberen Bereiche, erscheint ein Bestehen zwanzig enger Freundschaften bei einer einzelnen Person eher fragwürdig. Daher werden die Ausprägungen der Variable ab einer Anzahl von sechs engen Freunden in Kategorien zusammengefasst. 
Die Netzwerkheterogenität steigt jeweils um einen Skalenpunkt, wenn der Befragte 1. Freunde, 2. Helfer, 3. Vereinsmitgliedschaften oder 4. Haushaltsmitbewohner hat, 5. anderen hilft oder 6. mindestens flüchtigen Kontakt zu seinen Nachbarn pflegt. Der Heterogenitätsindex bewegt sich in einer Spanne von null bis sechs verfügbaren Netzwerkkomponenten und bezieht sich auf das Vorhandensein verschiedener „Informationspools“, erfasst so also auch eher schwächere Beziehungen. ${ }^{8}$

Tabelle 1: Überblick über verwendete Netzwerkvariablen

\begin{tabular}{l|l}
\hline Beziehungsarten & Beschreibung (Ausprägungen bei Erwerbslosen in Welle 3) \\
\hline Freunde & $\begin{array}{l}\text { Anzahl enger Freunde, im oberen Bereich klassifiziert } \\
(0,1,2,3,4,5,6-10=6,11+=7)\end{array}$ \\
\hline Haushaltsmitglieder & Anzahl der Haushaltsmitglieder > 15 Jahre ohne Ego (0-4) \\
\hline Helfer & $\begin{array}{l}\text { Durchschnittliche Anzahl der Helfer bzgl. verschiedener fiktiver } \\
\text { Hilfesituationen }(0-5)\end{array}$ \\
\hline Hilfe & $\begin{array}{l}\text { Anzahl der Personen, denen Ego in den vorangegangenen sechs Monaten } \\
\text { geholfen hat }(0-3)\end{array}$ \\
\hline Nachbarn & $\begin{array}{l}\text { Intensität der Nachbarschaftskontakte } \\
(0 \text { (kein Kontakt) }-4 \text { (sehr enger Kontakt)) }\end{array}$ \\
\hline Netzwerkeigenschaften & Vereinsmitgliedschaften im Haushalt (0-5) \\
\hline Netzwerkgröße & Anzahl der Freunde und Haushaltsmitglieder $(0-8)$ \\
\hline Netzwerkheterogenität & Anzahl der einzelnen Beziehungspools $(0-6)$ \\
\hline
\end{tabular}

b) Kontrollvariablen. Sowohl das Bestehen sozialer Beziehungen als auch die Erfolgsaussichten bei der Stellensuche werden von vielen Faktoren beeinflusst (z.B. Andreß 1989; Tazelaar 1990; Vishwanath 2001; Gilberg et al. 2001; Uhlendorff 2004). Daher werden einige der auf der Individualebene erhobenen Variablen im multivariaten Modell kontrolliert. ${ }^{9}$ Ein Überblick über alle Kontrollvariablen, ihre Zeitabhängigkeit sowie deren Berücksichtigung im Modell lässt sich aus Tabelle 2 gewinnen.

Veränderliche Merkmale (Schulbildung, Berufsausbildung, Gesundheitszustand, subjektives Haushaltsauskommen, Kinder) werden mit Ausnahme der retrospektiv erfragten Merkmale aus der jeweiligen Vorwelle übertragen, da sie ihren Einfluss zur Zeit der Suche ausüben. Ausprägungen in der aktuellen Welle könnten außerdem auch mit dem Finden eines Arbeitsplatzes in neuerer Zeit zu tun haben, der kausale Zusammenhang wird also wiederum zeitlich abgegrenzt.

8 Die Haushaltsgröße liegt für alle bis zur jeweiligen Welle im Panel verbliebenen Zielpersonen vor. Insgesamt unterschätzen beide Indizes die tatsächlichen Netzwerkausprägungen, da bei Nichtbeantwortung weiterer Fragen zu sozialen Beziehungen jeweils eine „0“ vergeben wird. Dies scheint treffender als eine Durchschnittsbildung aus anderen Beobachtungen, denn es ist davon auszugehen, dass die Nichtbeantwortung der Netzwerkfragen nicht zufällig stattfindet, sondern eventuell eben aus dem Nichtvorhandensein sozialer Beziehungen entsteht.

9 Auf strukturelle Bedingungen (regionale Arbeitslosenquoten, Arbeitsvermittlungsstruktur etc.) kann in diesem Rahmen nicht eingegangen werden. 
Tabelle 2: Überblick über die Kontrollvariablen und zeitliche Dimensionen

\begin{tabular}{|c|c|c|}
\hline Variable & Ausprägungen & Zeitliche Dimension \\
\hline \multicolumn{3}{|l|}{ Objektive Merkmale } \\
\hline Höchste Schulbildung & $=1$, wenn mittlere oder Hochschul-Reife & $\begin{array}{l}\text { Veränderlich } \\
\text { Übertrag aus Vorwelle }\end{array}$ \\
\hline Berufsausbildung & $=1$, wenn (abgeschlossene) Ausbildung & $\begin{array}{l}\text { Veränderlich } \\
\text { Übertrag aus Vorwelle }\end{array}$ \\
\hline $\begin{array}{l}\text { Erwerbslosigkeit vor } \\
\text { Welle } 3\end{array}$ & $\begin{array}{l}=1, \text { wenn keine Welle vor Welle } 3 \\
=1, \text { wenn eine Welle vor Welle } 3 \\
=1, \text { wenn zwei Wellen vor Welle } 3\end{array}$ & Konstant \\
\hline Gesundheitszustand & $=1$, wenn „sehr schlecht" oder „eher schlecht“ & $\begin{array}{l}\text { Veränderlich } \\
\text { Übertrag aus Vorwelle }\end{array}$ \\
\hline Alter & $16-64$ & $\begin{array}{l}\text { Zeitkontinuierliche } \\
\text { Veränderung }\end{array}$ \\
\hline Geschlecht & $=1$, wenn männlich & Konstant \\
\hline Staatsbürgerschaft & $=1$, wenn ausländ./doppelte Staatsangehörigkeit & Konstant \\
\hline \multicolumn{3}{|l|}{ Notwendigkeit } \\
\hline $\begin{array}{l}\text { Subjektives } \\
\text { Haushaltsauskommen }\end{array}$ & $=1$, wenn "schlecht“ & $\begin{array}{l}\text { Veränderlich } \\
\text { Übertrag aus Vorwelle }\end{array}$ \\
\hline \multicolumn{3}{|l|}{ Institutionelle Hilfen } \\
\hline Beratung durch Ämter & $\begin{array}{l}=1, \text { wenn Beratung/Ausbildung/Umschulung/ } \\
\text { Fortbildung durch Arbeits-/Sozialamt }\end{array}$ & $\begin{array}{l}\text { Veränderlich } \\
\text { retrospektiv erfragt }\end{array}$ \\
\hline \multicolumn{3}{|c|}{ Beeinträchtigung der zeitlichen Flexibilität } \\
\hline Kinder & $=1$, wenn Kind(er) im Haushalt & $\begin{array}{l}\text { Veränderlich } \\
\text { Übertrag aus Vorwelle }\end{array}$ \\
\hline $\begin{array}{l}\text { Belastende } \\
\text { Lebensumstände }\end{array}$ & $\begin{array}{l}=1, \text { wenn zu pflegende oder suchtkranke } \\
\text { Angehörige }\end{array}$ & $\begin{array}{l}\text { Veränderlich } \\
\text { aktuelle Nennung }\end{array}$ \\
\hline \multicolumn{3}{|l|}{ Kontext } \\
\hline Untersuchungsregion & $=1$, wenn Westdeutschland & $\begin{array}{l}\text { Veränderlich } \\
\text { aktuelle Nennung }\end{array}$ \\
\hline Erhebungszeitpunkt & $\begin{array}{l}=1, \text { wenn Welle } 4 \\
=1, \text { wenn Welle } 5 \\
=1, \text { wenn Welle } 6\end{array}$ & Zeitindikator \\
\hline
\end{tabular}

c) Untersuchungsgruppe. Als Untersuchungsgruppe werden alle Bezugspersonen des NIEP ausgewählt, die dem Arbeitsmarkt zur Verfügung stehen, jedoch nicht erwerbstätig sind und eine Erwerbstätigkeit wünschen. Erwerbslosigkeit wird definiert als Arbeitslosigkeit im engen Sinne einer Meldung als arbeitslos oder als Arbeitssuchender beim Arbeitsamt, aber auch als eigene Einstufung („arbeitslos“) bei der Frage nach Gründen des Nichterwerbsstatus. Die Berücksichtigung der Arbeitslosen nach Selbsteinstufung erfolgt, da die Meldung beim Arbeitsamt für viele Personen eine Hürde darstellt und hier alle Erwerbslosen erfasst werden sollen.

Die Auswahlkriterien treffen auf 296 Individuen in der dritten Welle zu. Sie verbleiben in Erwerbslosigkeit, werden erwerbsunfähig ${ }^{10}$ oder finden einen Arbeitsplatz,

10 Die konkurrierenden Zielzustände sind im Einzelnen: Alleinerziehend mit Kindern unter 3 
was von Welle zu Welle verfolgt wird, d.h. es werden drei mögliche Wechsel bis zum Ende des Panels in Welle 6 betrachtet.

Als erwerbstätig wird bezeichnet, wer angibt, einer Voll- oder Teilzeitbeschäftigung nachzugehen. Im Folgenden werden Personen, die nach Welle 3 einen Job finden, der Einfachheit halber als „Aussteiger" bezeichnet, während solche, die in Erwerbslosigkeit verbleiben, „Erwerbslose“ genannt werden.

In die multivariate Analyse geht jede erwerbslose Person mit allen Beobachtungen bis zu ihrer letzten Erwerbslosigkeitswelle oder der ersten Welle eines Abgangs in Erwerbstätigkeit ein. Mehrfachwechsel zwischen diesen beiden Zuständen werden nicht berücksichtigt. Die Einschränkung auf den ersten Abgang erfolgt erstens aufgrund der Tatsache, dass der Verbleib im Job mit hier nicht beobachteten Größen wie Motivation, Pünktlichkeit, Eignung für die Stelle, kollegialem Verhältnis etc. zusammenhängt. Zweitens muss von der Konstanz der Netzwerke ausgegangen werden, die bei der Betrachtung weiterer Ausstiege aus erneuter Erwerbslosigkeit nicht mehr gegeben wäre, da möglicherweise neue (Arbeits-)Kontakte nicht berücksichtigt werden könnten. ${ }^{11}$

\section{Die Analyse diskreter Ereignisdaten}

Um die Auswirkungen verschiedener Merkmale auf die Wahrscheinlichkeit des Ausstiegs aus Erwerbslosigkeit zu bestimmen, eignet sich die Ereignisdatenanalyse, da diese zulässt, mehrere aufeinander folgende Beobachtungen im „person-period-file“ und zeitveränderliche sowie zeitunabhängige Variablen zu berücksichtigen (vgl. Allison 1984). Bei dem hier zu untersuchenden Ereignis handelt es sich um den Übergang aus Erwerbslosigkeit in Erwerbstätigkeit, es findet also ein Wechsel zwischen zwei Zuständen statt, der zu bestimmten Zeitpunkten erhoben wurde. Im Folgenden wird daher eine Methode zur Analyse diskreter Ereignisdaten angewendet (vgl. z.B. Allison 1984: 17f.; Yamaguchi 1991: 18f.; Andreß 1992a: 271f.).

Es lässt sich zeigen, dass die bedingte Wahrscheinlichkeit eines Übergangs bei singulären Ereignissen in ein Logit-Modell übersetzt werden kann; es gleicht bis auf die Einführung der Zeit einer einfachen logistischen Regression. Die zeitbedingten LogOdds werden bestimmt als linear-additive Funktion der zeitabhängigen Konstanten und der einzelnen Effekte zeitabhängiger und zeitunabhängiger Kovariaten, die simultan im Modell berücksichtigt werden können (Allison 1984: 17ff.). Eine mögliche Veränderung der Chancen je Erhebungszeitpunkt wird mithilfe von Indikator-Variablen für die Befragungswellen ins Modell einbezogen (Allison 1984: 19f.).

Hier wird nur ein Zielzustand - die Aufnahme einer Erwerbstätigkeit - betrachtet, da man zeigen kann, dass einzelne logistische Regressionen für verschiedene konkurrierende Zielzustände ähnliche Ergebnisse liefern, wie eine multinomiale logistische Regression, die alle möglichen Zielzustände gleichzeitig berücksichtigt (Allison 1984: 46f.). Zusätzlich verlangt die Berücksichtigung mehrerer Beobachtungen pro Person bei der Auswertung von Daten über singuläre Ereignisse unter Verwendung der Maximum-

Jahren, Rentner und auf Rente Wartende, längere Krankheit und Pflege Angehöriger ohne Unterstützung.

11 Eine ausführlichere Beschreibung der Untersuchungsgruppe findet sich in Brandt (2004). 
Likelihood-Schätzung keine Kontrolle unbeobachteter Heterogenität (Allison 1995: 223f.). Die Frage, die mit den folgenden Auswertungen beantwortet werden soll, bezieht sich auf die Abgangsrate bzw. die Chance eines Ausstiegs unter der Berücksichtigung von zeitlichen Veränderungen.

\section{Befunde}

1. Soziale Beziehungen und Wege der Stellenfindung

Die im folgenden Abschnitt betrachtete Risikopopulation setzt sich aus 222 in Welle 3 erwerbslosen Personen zusammen. Über die gewählte Sequenz von Welle 4 bis Welle 6 ergeben sich 475 Beobachtungen bei 82 Aussteigern (37 Prozent) und 140 erwerbslos Verbleibenden (63 Prozent).

Tabelle 3 gibt einen Überblick über die Verteilung von Persönlichkeits- und Kontextmerkmalen in der Untersuchungsgruppe, auch aufgeschlüsselt nach Erfolgreichen und Erfolglosen.

Tabelle 3: Verteilung der kontrollierten Eigenschaften (Prozent $/{ }^{\circ}$ Arithm. Mittel)

\begin{tabular}{l|c|c|c}
\hline & Aussteiger $(\mathrm{n}=82)$ & Erwerbslose $(\mathrm{n}=140)$ & Gesamt $(\mathrm{n}=222)$ \\
\hline Mittlere \& Hochschulreife & 59,0 & 60,7 & 60,2 \\
Abgeschlossene Ausbildung & 93,9 & 78,6 & 83,0 \\
Eine/zwei Welle(n) erwerbslos vor & $16,2 /$ & $27,3 /$ & $24,1 /$ \\
Welle 3 & 62,4 & 67,5 & 66,1 \\
Schlechter Gesundheitszustand & 4,6 & 20,3 & 15,8 \\
Alter (24-63) & 44,5 & $\circ 5,5$ & 45,2 \\
Mann & 37,6 & 44,6 & 42,5 \\
Ausländer & 5,7 & 2,4 & 3,4 \\
Schlechtes Haushaltsauskommen & 49,7 & 67,4 & 62,3 \\
Beratung durch Ämter & 15,4 & 34,2 & 28,8 \\
Kinder & 47,9 & 34,3 & 38,1 \\
Belastende Lebensumstände & 9,6 & 8,3 & 8,7 \\
Westdeutschland & 55,2 & 49,9 & 51,4 \\
\hline
\end{tabular}

Datenbasis: NIEP, gewichtete Ergebnisse, eigene Berechnungen.

Die zu 100 fehlenden Prozent entfallen jeweils auf die Referenzkategorie.

Gliedert man auch die Netzwerkindizes, wie in den Abbildungen 1 und 2 dargestellt, auf Aussteiger und Erwerbslose auf, lassen sich deutliche Unterschiede zwischen den Gruppen erkennen:

Aussteiger haben größere Netzwerke als Erwerbslose. Mehr als die Hälfte der Erwerbslosen hat ein Netzwerk mit keinem bis zwei engen Freunden und/oder Haushaltsmitgliedern (Median = 2). Dagegen sind Aussteiger ab einer Größe von drei bis zu einer Größe von sieben insgesamt stärker vertreten. Die mittleren beiden Quartile verteilen sich bei Letzteren zwischen zwei und vier Kontaktpartnern (Median = 3), am häufigsten besitzen sie vier enge Kontakte.

Für die Heterogenität lässt sich nach Abbildung 2 Ähnliches feststellen: Erwerbslose haben häufiger nur zwei und drei Netzwerkbestandteile als solche, die im weiteren Verlauf den Ausstieg schaffen, bei vier nähern sich die Gruppen an. Aussteiger verfügen zu 
Abbildung 1: Netzwerkgröße bei Aussteigern und Erwerbslosen

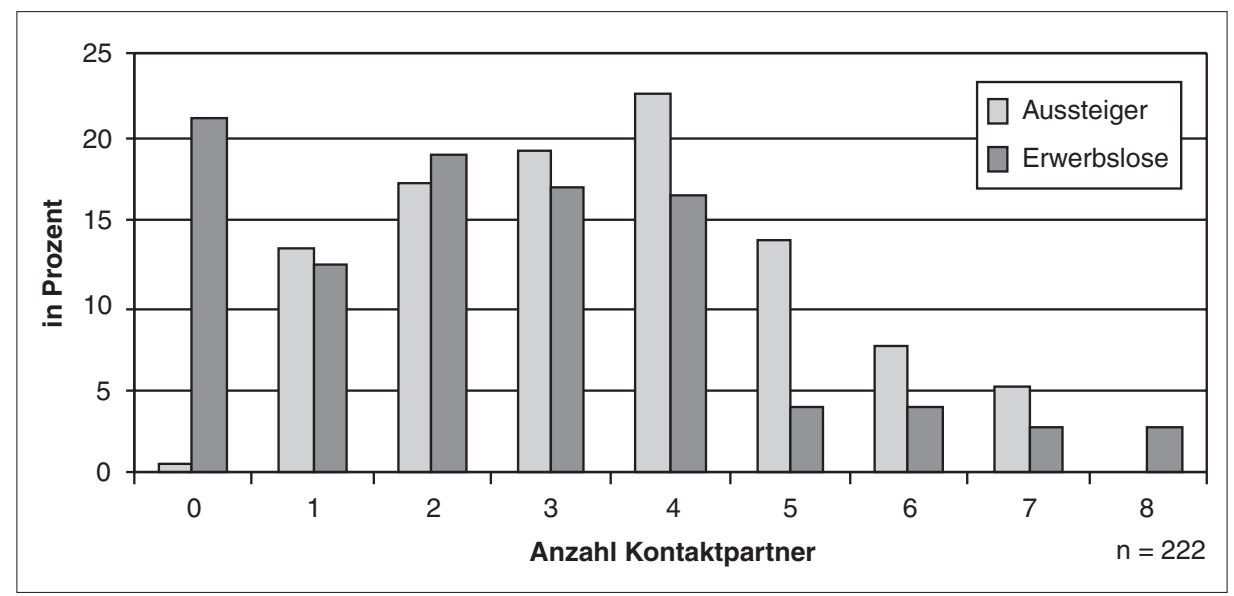

Mittelwert je Gruppe: Aussteiger $=3,48 /$ Erwerbslose $=2,54$.

Datenbasis: NIEP, gewichtete Ergebnisse, eigene Berechnungen.

ca. 45 Prozent über fünf verschiedene Kontaktpools und haben insgesamt deutlich häufiger fünf oder sechs verschiedene Netzwerkkomponenten als Erwerbslose.

Sowohl die Quantität der (engen) Kontaktpersonen als auch die Ausdehnung des Netzwerks auf unterschiedliche Beziehungsarten zeigen also einen klaren Zusammenhang mit dem Ereignis eines Ausstiegs aus Erwerbslosigkeit im Niedrigeinkommensbereich.

Abbildung 2: Netzwerkheterogenität bei Aussteigern und Erwerbslosen

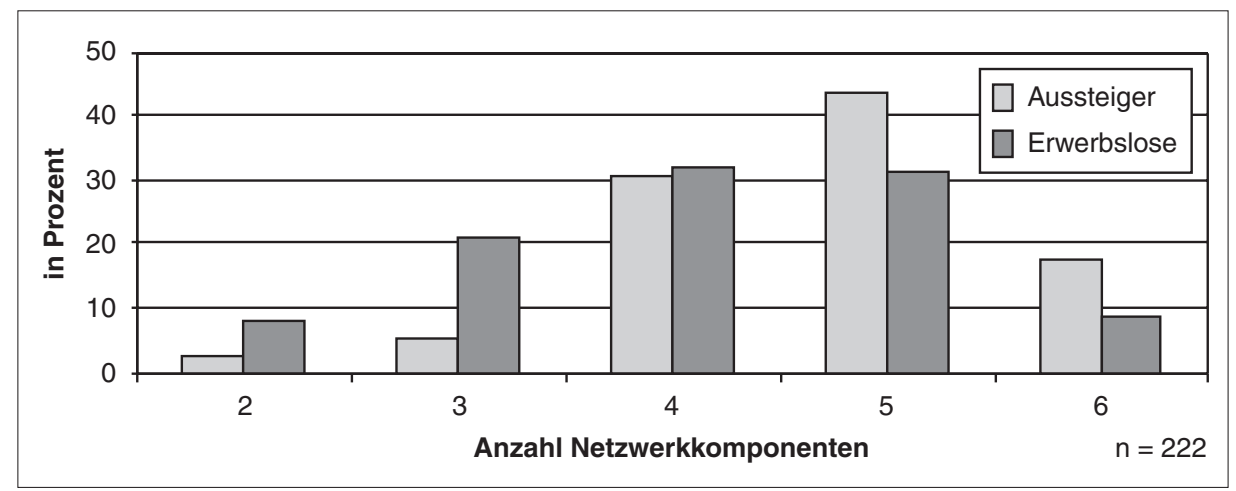

Mittelwert je Gruppe: Aussteiger = 4,69 / Erwerbslose $=4,12$.

Datenbasis: NIEP, gewichtete Ergebnisse, eigene Berechnungen. 
29 Prozent der Aussteiger ( $\mathrm{n}=76$; weitere Wege: Ämter 34 Prozent, Anzeige/Blindbewerbung/private Stellenvermittlung 35 Prozent, andere 2 Prozent), geben an, die neue Stelle mithilfe sozialer Beziehungen gefunden zu haben. ${ }^{12}$ Das Zwischenergebnis stützt die beschriebenen Forschungsergebnisse. Es lässt sich also festhalten: Die Nutzung sozialer Kontakte verbessert die Erfolgsaussichten von Niedrigeinkommensbeziehern bei der Stellensuche. (H1)

Die folgende Analyse stützt sich auf dieses Ergebnis und soll einzelne Netzwerkeigenschaften und Randbedingungen für diese Ereignisse in ihrem Zusammenwirken testen.

2. Der Effekt sozialer Beziehungen auf die Ausstiegschance

Um den Einfluss sozialer Netzwerke auf die Ausstiegschance zu analysieren, werden zunächst beide Netzwerkindikatoren einzeln ohne Berücksichtigung anderer möglicher Einflüsse überprüft. Beide Modelle sind nach dem LR-Test signifikant, Modell 1, das die Netzwerkheterogenität berücksichtigt, weist jedoch die höhere Modellgüte auf $\left(\mathrm{BIC}(\mathrm{M} 1)=\operatorname{BIC}(\mathrm{M} 2)-2,82\right.$; bzw. wg. $\mathrm{k}(\mathrm{M} 1, \mathrm{M} 2)=1$ : LR $\chi^{2}$ (M1): 10,51*** LR $\chi^{2}$ (M2): 7,69**).

In den Abbildungen 3 und 4 werden die vorhergesagten Wahrscheinlichkeiten mit den beobachteten Wahrscheinlichkeiten verglichen. Die erste Abbildung zeigt den prognostizierten Einfluss der Netzwerkheterogenität auf die Ausstiegswahrscheinlichkeit im Gegensatz zu den tatsächlichen Ausstiegen von Personen mit entsprechendem Netzwerk. Eine Zunahme der Netzwerkheterogenität um eine Einheit bewirkt eine signifikante Erhöhung des Odds Ratios. Somit steigt die Chance, einen Ausstieg aus Erwerbslosigkeit zu schaffen, mit der Anzahl der Netzwerkkomponenten.

Der im Modell berechnete Zusammenhang zwischen der Zahl der Netzwerkkomponenten und der Ausstiegschance beschreibt wie in Abbildung 3 ersichtlich die Realität in der Untersuchungsgruppe weitgehend richtig.

Eine Vergrößerung des Netzwerks um eins bewirkt dagegen eine geringere, aber ebenfalls signifikante Verbesserung der Ausstiegschance. Jeder zusätzliche enge Freund oder jedes erwachsene Haushaltsmitglied erhöht nach Modell 2 die Chance, einen Ausstieg aus Erwerbslosigkeit zu schaffen. Personen ohne enge Kontaktpartner haben eine Ausstiegschance von knapp 0,1. In der nächsten Abbildung wird analog die in Modell 2 geschätzte und die beobachtete Ausstiegswahrscheinlichkeit in Abhängigkeit von der Netzwerkgröße dargestellt (Funktion mit Rechtecken): Es zeigt sich deutlich, dass die Prognose anhand des Modells 2 sowohl bei einer Netzwerkgröße von Null als auch ab

12 Die zuvor (nicht unbedingt erfolgreich) genutzten Suchwege wurden nur bei offiziell arbeitslos gemeldeten Personen und Sozialhilfeempfängern abgefragt, können also für die hier betrachtete Gruppe aller in Welle 3 Erwerbslosen nicht angegeben werden. Eine tendenzielle Einschätzung erlauben aber die Ergebnisse, die Andreß und Krüger (2006: 44f.) auf Basis eines anderen Samples aus dem NIEP veröffentlicht haben. Weitere Ergebnisse zum Verhältnis von Suchwegen und (auch finanziellem) Erfolg aufgrund anderer Daten (auch Jobwechsler) bieten z.B. Uhlendorff (2004), Noll und Weick (2002), Pischner et al. (2002) und Grund (2001). 
Abbildung 3: Einfluss der Netzwerkheterogenität. Beobachtete / prognostizierte Ausstiegswahrscheinlichkeit und Netzwerkheterogenität (M1)

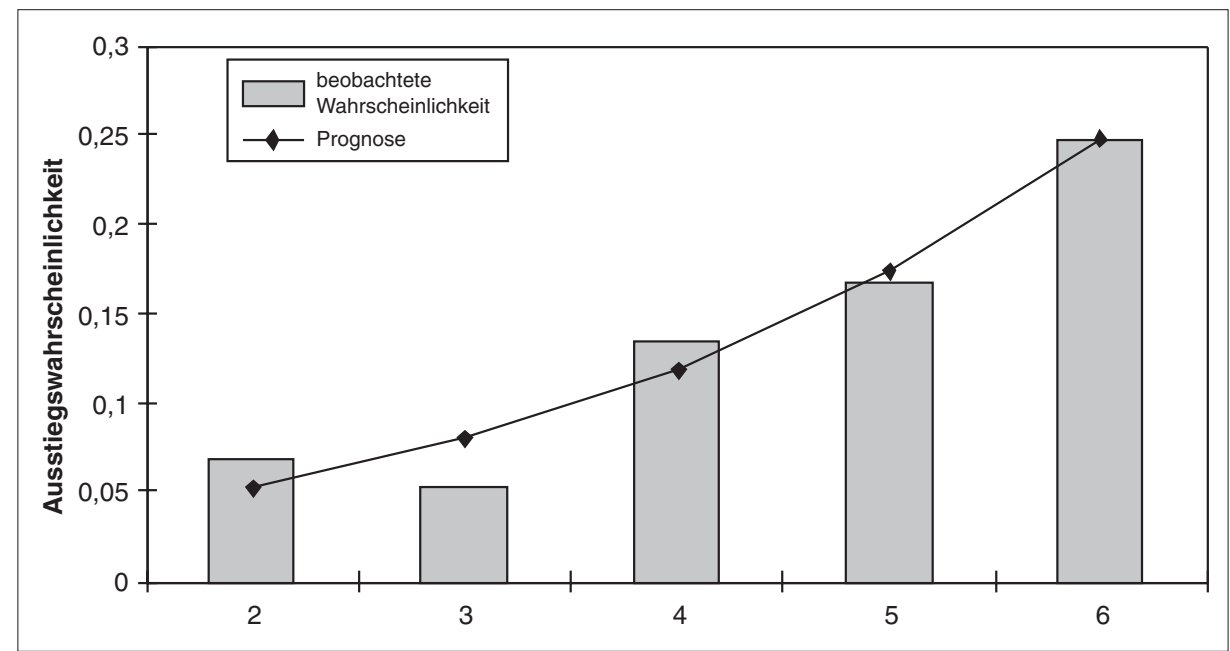

$\mathrm{OR}=1,56^{* * *} ;$ Koeff. $=0,45 ; \mathrm{SE}=0,14 ; \mathrm{n}=222,475$ Beob.

Datenbasis: NIEP, gewichtet Ergebnisse, eigene Berechnungen SE = Standardfehler.

(Effekt-)Koeffizienten (OR) signifikant zum ${ }^{*}<0,1^{* *}<0,05^{* * *}<0,01$-Niveau.

Abbildung 4: Einfluss der Netzwerkgröße. Beobachtete / prognostizierte Ausstiegswahrscheinlichkeit und Netzwerkgröße (M2 und M3)

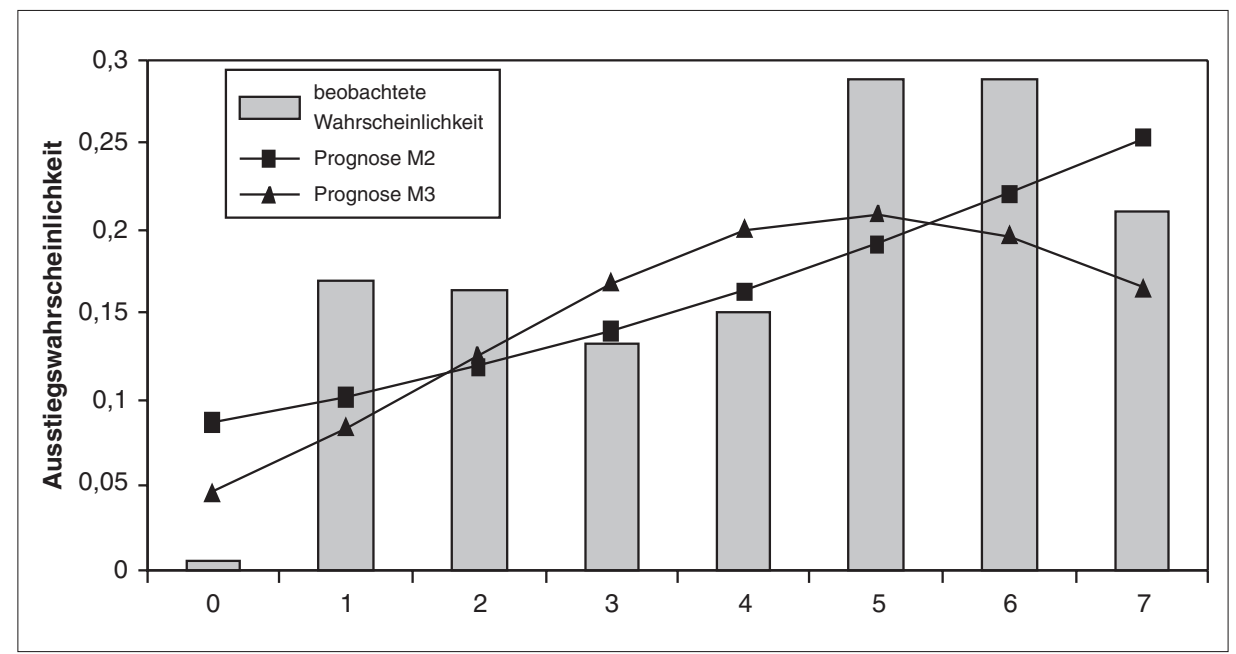

M2: $\mathrm{OR}=1,20^{* * *} ;$ Koeff. $=0,18, \mathrm{SE}=0,07$.

M3: OR $=1,98^{* * *} / 0,93^{* *}$; Koeff. $=0,68 /-0,07, \mathrm{SE}=0,23 / 0,03$.

$\mathrm{n}=222,475$ Beob.

Datenbasis: NIEP, gewichtete Ergebnisse, eigene Berechnungen. SE = Standardfehler.

(Effekt-)Koeffizienten (OR) signifikant zum ${ }^{*}<0,1,{ }^{* *}<0,05$ und ${ }^{* * *}<0,01$-Niveau. 
einer Netzwerkgröße von sieben die tatsächlich beobachteten Wahrscheinlichkeiten übertrifft.

Nach den beobachteten Werten scheint in der Untersuchungsgruppe vor allem die Existenz eines (Freundes- und Haushalts-)Netzwerks an sich einen positiven Einfluss auf die Ausstiegswahrscheinlichkeit zu haben. Dieser vergrößert sich unregelmäßig bis zu einer Größe von sechs; ab der Ausprägung sieben sinkt die beobachtete Wahrscheinlichkeit wieder, einen Ausstieg zu schaffen. Es scheint also nicht nur einen Sättigungspunkt zu geben, ab dem mehr Kontakte nicht mehr Wirkung zeigen, sondern sogar einen abnehmenden Effekt. Die Einführung eines quadratischen Effekts führt im vorliegenden Fall zu einer signifikanten Modellverbesserung ( $\mathrm{LR} \chi^{2}=5.87^{* *} \mid \mathrm{k}=1$ ). Die Prognose, die dann erfolgt, ist in der Abbildung mit der umgekehrt U-förmigen Funktion (Modell 3) abgetragen.

Bei einer Netzwerkgröße von eins besteht im Vergleich zu einer Netzwerkgröße von null eine erhöhte Chance, einen Ausstieg aus Erwerbslosigkeit zu vollbringen. Die höchste Chance für einen Ausstieg besteht bei fünf Netzwerkpartnern, darüber sinkt die Chance laut Schätzung wieder ab.

Es zeigt sich also, dass die Anzahl der (engen) Kontaktpartner nur bis zu einer gewissen Anzahl von steigendem Nutzen für die Stellensuche ist, was daran liegen könnte, dass Personen mit mehr Freunden und Haushaltsangehörigen durch ihre starken und somit restriktiven Netzwerkverpflichtungen so in Anspruch genommen werden, dass eine Jobsuche eher erschwert wird.

$\mathrm{Ob}$ sich der in den drei Modellen festgestellte Effekt sozialer Beziehungen auch unter Kontrolle der üblicherweise zur Analyse des Ausstiegs aus Arbeitslosigkeit verwendeten Faktoren und unter expliziter Berücksichtigung der zeitlichen Abhängigkeit bestätigen lässt, wird in den Modellen Modell 4 und Modell 5 (Tabelle 4) geprüft. Beide Netzwerkmerkmale stehen inhaltlich in starkem Zusammenhang: Wer zu mehr Menschen eine soziale Beziehung unterhält, kennt auch eher Menschen aus mehreren unterschiedlichen Kreisen. Daher werden die Komponenten einzeln im ansonsten identischen Modell getestet, um dem Problem der Ähnlichkeit ihrer Effekte aus dem Weg zu gehen und eine signifikante Aussage über jeden Faktor einzeln machen zu können. ${ }^{13}$

Auch unter Kontrolle der beschriebenen Dritteinflüsse ist laut Modell 4 ein Effekt der Heterogenität festzustellen, der sowohl in seiner Höhe als auch in seiner Signifikanz dem im restringierten Modell (Modell 1) geschätzten Effekt ähnelt. Im Vergleich der Modelle erzeugt die Einführung der Drittvariablen nach dem LR-Test eine signifikante Modellverbesserung ( $\mathrm{LR} \chi^{2}=50,17^{* * *} \mid \mathrm{k}=15$ ). Dasselbe ist auch in den rechten Spalten feststellbar, in denen das fünfte Modell mit der Netzwerkgröße abgetragen ist. Der Effekt der Netzwerkgröße hat sich im Vergleich zum restringierten Modell 3 vergrößert, die Einführung der Drittvariablen verbessert die Modellgüte signifikant (LR $\left.\chi^{2}=55,49^{* * *} \mid \mathrm{k}=15\right)$.

In beiden Modellen zeigen sich sehr ähnliche Effekte der Drittvariablen, was für die

13 Der variance inflation factor ist zwar nicht bedenklich hoch, wenn man beide Netzwerkindikatoren im selben Modell berücksichtigt, es zeigt sich aber in Kontrollrechnungen, dass auf diese Weise für die Netzwerkeigenschaften keine stabilen Effekte mehr geschätzt werden können. In allen weiteren Kontrollrechnungen (u.a. Ausschluss einflussreicher Fälle, robuste Standardfehler, random effects) zeigen sich die Netzwerkindikatoren stabil signifikant positiv. 
Tabelle 4: Heterogenität und Dritteinflüsse (Modell 4), Größe ${ }^{2}$ und Dritteinflüsse (Modell 5)

\begin{tabular}{|c|c|c|c|c|c|c|}
\hline & Odds Ratio & Koeffizient & SE & Odds Ratio & Koeffizient & SE \\
\hline Netzwerkheterogenität & $1,43^{* *}$ & 0,35 & 0,17 & - & - & - \\
\hline Netzwerkgröße & - & - & - & $2,31^{* * *}$ & 0,84 & 0,28 \\
\hline Netzwerkgröße ${ }^{2}$ & - & - & - & $0,92^{* *}$ & $-0,09$ & 0,04 \\
\hline Mittlere \& Hochschulreife & 0,69 & $-0,37$ & 0,33 & 0,69 & $-0,38$ & 0,33 \\
\hline Abgeschlossene Ausbildung & 2,21 & 0,79 & 0,59 & $2,74^{*}$ & 1,01 & 0,59 \\
\hline $\begin{array}{l}\text { Keine Erwerbslosigkeit vor } \\
\text { Welle } 3\end{array}$ & \multicolumn{6}{|c|}{ Referenz } \\
\hline Erwerbslosigkeit (eine Welle) & $0,24^{* * *}$ & $-1,42$ & 0,52 & $0,22^{* * *}$ & $-1,52$ & 0,53 \\
\hline Erwerbslosigkeit (zwei Wellen) & $0,28^{* * *}$ & $-1,26$ & 0,43 & $0,26^{* * *}$ & $-1,36$ & 0,45 \\
\hline Schlechte Gesundheit & $0,20^{* *}$ & $-1,6$ & 0,64 & $0,21^{* *}$ & $-1,58$ & 0,64 \\
\hline Alter & 0,97 & $-0,03$ & 0,02 & 0,99 & $-0,01$ & 0,02 \\
\hline Mann & 0,88 & $-0,13$ & 0,34 & 0,70 & $-0,36$ & 0,35 \\
\hline Ausländer & 1,32 & 0,27 & 0,66 & 1,18 & 0,16 & 0,67 \\
\hline $\begin{array}{l}\text { Schlechtes Haushalts- } \\
\text { auskommen }\end{array}$ & 0,66 & $-0,41$ & 0,31 & 0,73 & $-0,31$ & 0,32 \\
\hline Beratung durch Ämter & $0,26^{* * *}$ & $-1,36$ & 0,38 & $0,23^{* * *}$ & $-1,47$ & 0,38 \\
\hline Kinder & 0,85 & $-0,16$ & 0,38 & 0,96 & 0,04 & 0,38 \\
\hline Belastende Lebensumstände & 0,63 & $-0,46$ & 0,54 & 0,90 & $-0,10$ & 0,56 \\
\hline Westdeutschland & 1,33 & 0,29 & 0,31 & 1,38 & 0,32 & 0,32 \\
\hline Welle 4 & \multicolumn{6}{|c|}{ Referenz } \\
\hline Welle 5 & $2,02^{* *}$ & 0,71 & 0,33 & $2,04^{* *}$ & 0,71 & 0,33 \\
\hline Welle 6 & 1,36 & 0,30 & 0,41 & 1,40 & 0,33 & 0,41 \\
\hline Konstante & - & $-0,84$ & 1,43 & - & $-1,90$ & 1,46 \\
\hline Pseudo R² (Mc Fadden) & & 0,16 & & & 0,18 & \\
\hline LogLikelihood & & $-165,18$ & & & $-161,00$ & \\
\hline LR $\chi^{2}(\mathrm{k}=16 \mid \mathrm{k}=17)$ & & $60,69^{* * *}$ & & & $69,06^{* * *}$ & \\
\hline $\mathrm{N}=222$ & & 475 Beob. & & & 475 Beob. & \\
\hline
\end{tabular}

Datenbasis: NIEP, gewichtete Ergebnisse, eigene Berechnungen.

$\mathrm{SE}=$ Standardfehler. (Effekt-) Koeffizient signifikant zum ${ }^{*}<0,1,{ }^{* *}<0,05$ und ${ }^{* * *}<0,01$-Niveau.

Stabilität ihrer hier gemessenen Wirkung und auch für ihre Unabhängigkeit vom verwendeten Netzwerkkonzept spricht. Stark negative signifikante Effekte haben in beiden Modellen vor allem der Gesundheitszustand in der Vorwelle, die Erwerbslosigkeitsdauer im Panel und die vergangene Beratung durch Ämter.

Letzteres ist bei einer Zahl von 34 Prozent, die angeben, ihre Stelle über das Arbeitsamt gefunden zu haben, zunächst ein überraschendes Ergebnis. Es ist aber plausibel, wenn man davon ausgeht, dass - zusätzlich zur eingeschränkten Wirksamkeit oder nicht stattfindender Umsetzung der in der Beratung empfohlenen Maßnahmen - vor allem diejenigen, bei denen die Vermittlung über das Amt zuvor gescheitert ist, eine Beratung zu Ausbildung, Umschulung oder Fortbildung vom Arbeits- oder Sozialamt 
in Anspruch nehmen. ${ }^{14}$ Das sind also vermutlich Personen, die besonders schlechte (in diesem Modell nicht kontrollierte) Voraussetzungen mitbringen.

Während die erste im Panel vor Welle 3 in Erwerbslosigkeit verbrachte Welle die Chance auf einen Ausstieg stark senkt, scheint dieser Effekt mit der Zeit (bzw. der zweiten Erwerbslosigkeitswelle) zumindest nicht weiter anzusteigen (siehe auch Uhlendorff 2004: 296). ${ }^{15}$ Besonders eine längere (d.h. hier mehr als den Befragungszeitpunkt in Welle 3 andauernde) Erwerbslosigkeit an sich hat also einen negativen Einfluss wobei an dieser Stelle einschränkend angemerkt werden muss, dass der hier untersuchte Zeitraum nicht ausreicht, um gültige Aussagen über Dauerarbeitslosigkeit zu machen.

Eine Erhöhung der Chance auf einen Ausstieg besteht in Welle 5 im Gegensatz zu Welle 4, möglicherweise wirkt sich die Verschlechterung der Arbeitsmarktlage in der vierten und sechsten Welle und ihre kurze (saisonale) Entspannung im Verlauf der fünften Welle (Juli-Oktober 2001) hier aus. Die Verbesserung der Chance zu diesem Zeitpunkt deutet aber auf weitere, nicht zu identifizierende Umstände wie über die Zeit vermittelte Kontextbedingungen (z.B. eine Änderung der Vermittlungsstruktur) hin. Ein zeitlicher Trend kann auch hier aufgrund der kurzen Untersuchungsdauer (Rechts- und Linkszensierung des Panels) an dem Ergebnis nicht abgelesen werden.

Bis auf die abgeschlossene Ausbildung, die in Modell 5 eine knapp signifikant höhere Chance auf den Ausstieg bewirkt, hat keine weitere objektive Eigenschaft einen signifikanten Einfluss. Für Kriterien wie Alter oder Geschlecht, oder auch für die Differenz zwischen der ost- und westdeutschen Arbeitsmarktlage, kann in den vorangehenden Modellen erstaunlicherweise kein signifikanter Effekt auf die Ausstiegschance festgestellt werden.

Umso deutlicher und überzeugender wird der stabile signifikante Einfluss, den soziale Beziehungen bzw. ihre hier gemessenen Eigenschaften bei Niedrigeinkommensbeziehern auf die Ausstiegschance haben: Steigt die Netzwerkheterogenität, steigt auch die Chance eines Ausstiegs signifikant (Modell 4).

Die Anzahl der Netzwerkpersonen (Modell 5) erhöht unter Annahme eines in den Extrembereichen abnehmenden Effekts und unter Kontrolle der beschriebenen Drittvariablen die Chance einer Stellenfindung ebenfalls. Ihr Einfluss steigt wie in Modell 3 bis zu einer Netzwerkgröße von fünf Personen an und sinkt dann wieder ab.

Die mithilfe eines BIC-Tests ermittelte Passgenauigkeit des Modells 5 zu den Daten

14 Für eine kritische Diskussion zur (Dokumentation der) Vermittlungstätigkeit des Arbeitsamts siehe Pischner et al. (2002).

15 Es wurde oben darauf hingewiesen, dass soziale Kontakte selbst von der Erwerbslosigkeitshistorie abhängen - eine Tatsache, die hier aufgrund der Linkszensierung des Panels (Informationen über die Dauer einer Erwerbslosigkeit nicht offiziell arbeitslos gemeldeter Personen vor Panelbeginn fehlen) nicht genauer kontrolliert werden kann. In Kontrollrechnungen zeigt sich jedoch: a) Bei Auswahl derjenigen Personen, für die die Arbeitslosigkeitsdauer retrospektiv (in Welle 4: Monate seit 1995) vorliegt, hat die Netzwerkgröße unter Kontrolle der Arbeitslosigkeitsdauer einen starken, signifikant positiven Effekt. Die Heterogenität wirkt sich in der Stichprobe weiterhin positiv aus, der Effekt verliert aber seine Signifikanz. b) Bei Auswahl der erst in Welle 3 Erwerbslosen gehen nur noch 74 Sequenzen in die Analyse ein, es können keine signifikanten Effekte mehr geschätzt und die Auswirkungen einer längeren Erwerbslosigkeit gar nicht mehr geprüft werden. 
ist leicht höher als bei Modell $4(\mathrm{BIC}(M 5)=\operatorname{BIC}(M 4)-2,21)$. Die Ergebnisse der ersten Modelle bestätigen sich also unter Berücksichtigung von Persönlichkeitsmerkmalen und Randbedingungen durchgängig. Es zeigt sich ein signifikanter Einfluss der überprüften Netzwerkeigenschaften, der auch unter Kontrolle von Humankapital, Gesundheitszustand, Erwerbslosigkeitshistorie und Belastungen des Suchenden gilt.

Nachdem die Bedeutung sozialer Beziehungen als Weg der Stellenfindung in der Stichprobe festgestellt wurde, halten auch die weiteren theoretisch hergeleiteten und auf Niedrigeinkommensbezieher übertragenen Hypothesen - allerdings mit einer Anpassung - der empirischen Überprüfung stand. Die Anzahl der engen Freunde und Haushaltsmitglieder hat einen signifikanten positiven Einfluss auf die Ausstiegschance. Bei sehr vielen Kontaktpartnern nimmt dieser Einfluss jedoch wieder ab. Es gilt also: Je größer das Netzwerk des Einzelnen ist, desto höher ist die Wahrscheinlichkeit eines Ausstiegs aus Erwerbslosigkeit (H2) - unter der Einschränkung, dass nur bis zu 5 engen Kontaktpartnern, d.h. eher starken Beziehungen eine Verbesserung der Chancen stattfindet. Auch die Netzwerkheterogenität, die die eher schwächeren Beziehungen beinhaltet, besitzt einen wichtigen und konstanten positiven Einfluss auf den möglichen Ausstieg aus Erwerbslosigkeit: Je heterogener das Netzwerk des Einzelnen ist, desto höher ist die Wahrscheinlichkeit eines Ausstiegs aus Erwerbslosigkeit (H3).

\section{Zusammenfassung}

Ausgehend von der "Strength of Weak Ties“-These (Granovetter 1973) wurde geprüft, ob soziale Beziehungen auch erwerbslosen Niedrigeinkommensbeziehern Ressourcen für die Stellensuche bereitstellen, d.h. Informationen und Einflussmöglichkeiten transportieren, die wichtig für die erfolgreiche Stellensuche sind. Zwei Eigenschaften von Beziehungsnetzen wurden besonders thematisiert: Die Vielfalt der im Netzwerk enthaltenen Beziehungsarten und die Anzahl der (engen) Netzwerkpartner.

Unter Berücksichtigung verschiedener Drittvariablen zeigt sich insgesamt ein stabiler positiver Einfluss großer und heterogener Netzwerke. Dies ist ein besonders bemerkenswertes Ergebnis, wenn man berücksichtigt, dass in anderen multivariaten Untersuchungen je Untersuchungsgruppe und Operationalisierung der Einfluss von Beziehungen durch Kontrollvariablen „überdeckt" wird (z.B. Wegener 1989: 291), nur eingeschränkte Ausmaße hat (z.B. Uhlendorff 2004: 298f.) oder dass soziales Kapital als intervenierende Variable betrachtet wird, die nur in Kombination mit Humankapital Wirkung zeigt (vgl. z.B. Runia 2002b: 26ff.).

Durchgängig positiv und steigend zeigt sich der Einfluss der verschiedenen im Netzwerk enthaltenen Netzwerkkomponenten. Das unterstützt die „Weak-Ties“-These Granovetters (1973), da die Heterogenitätsmessung in diesem Fall auch die potenziell schwachen Bindungen der Jobsuchenden beinhaltet. Wichtig für Informationsvorteile ist jedoch vor allem die Verschiedenartigkeit der sozialen Kontakte, die im Einzelnen ebenso stark wie schwach sein können.

Die Bedeutung starker Beziehungen als Brücken (z.B. Bian 1997) lässt sich mithilfe der Netzwerkgröße, die sich vor allem auf stärkere Beziehungen stützt, ebenfalls implizit nachweisen. Bis zu fünf engen Kontakten gilt: Wer ein größeres Netzwerk hat, fin- 
det eher eine Stelle. Zahlreiche Kontaktpartner verbessern die Chance auf einen Ausstieg aus der Erwerbslosigkeit, d.h. je mehr Kontakte, desto mehr Zugang zu wichtigen Informationen und Einflussmöglichkeiten bei der Jobsuche, außerdem dient ein umfangreiches Netzwerk als soziales Zeugnis beim Arbeitgeber (Lin 2001). Dies gilt auch bei Niedrigeinkommensbeziehern, und zwar unter Kontrolle von Kontexteinflüssen, Humankapital, Persönlichkeitsmerkmalen, Erwerbslosigkeitsdauer, institutionellen Hilfen und einschränkenden Lebensumständen.

Die Annahme, dass soziale Kontakte auch für die häufig aus Theorie und Empirie ausgeschlossenen erwerbslosen und ökonomisch schlecht gestellten Personen äußerst hilfreich für die Stellensuche und -findung sein können, bestätigt sich. Diese Analyse ergänzt die in vielen Untersuchungen als für den Ausstieg aus Erwerbslosigkeit sehr wichtig identifizierten „objektiven“ Einflüsse wie Geschlecht, Alter, Humankapital, Gesundheitszustand und Erwerbslosigkeitsdauer um einen wichtigen Einflussfaktor: soziale Kontakte.

\section{Literatur}

Allison, Paul D., 1984: Event History Analysis. Regression for Longitudinal Event Data. Sage University Paper Series on Quantitative Sciences 07-046. Beverly Hills/London: Sage.

Allison, Paul D., 1995: Survival Analysis Using SAS. A Practical Guide. Cary, NC: SAS Publishing.

Andreß, Hans-Jürgen, 1989: Instabile Karrieren und Erwerbslosigkeit. Ein Vergleich mit der Problemgruppe der Langzeitarbeitslosen - Theorien, Daten und einige explorative Ergebnisse. Mitteilungen aus der Arbeitsmarkt- und Berufsforschung 22: 17-32.

Andreß, Hans-Jürgen, 1992a: Einführung in die Verlaufsdatenanalyse. Statistische Grundlagen und Anwendungsbeispiele zur Längsschnittanalyse kategorialer Daten. Historical Social Research Supplement 5. Köln: Zentrum für Historische Sozialforschung.

Andreß, Hans-Jürgen, 1992b: Logistische Regressionsmodelle für Paneldaten. Analyse dichotomer Variablen im Zeitverlauf unter besonderer Berücksichtigung unbeobachteter Heterogenität. S. 35-66 in: Hans-Jürgen Andreß, Johannes Huinink, Holger Meinken, Dorothea Rumianek, Wolfgang Sodeur und Gabriele Sturm (Hg.), Theorie Daten Methoden. Neue Modelle und Verfahrensweisen in den Sozialwissenschaften. München: Oldenbourg.

Andreß, Hans-Jürgen, Gero Lipsmeier und Kurt Salentin, 1995: Soziale Isolation und mangelnde soziale Unterstützung im unteren Einkommensbereich? Vergleichende Analysen mit Umfragedaten. Zeitschrift für Soziologie 24: 300-315.

Andreß, Hans-Jürgen, und Anne Krüger, 2006: Ausstiege aus dem unteren Einkommensbereich. Institutionelle Hilfeangebote, individuelle Aktivitäten und soziale Netzwerke. Berlin: Edition Sigma.

Bian, Yanjie, 1997: Bringing Strong Ties Back. Indirect Ties, Network Bridges and Job Searches in China. American Sociological Review 62: 366-385.

Blaschke, Dieter, 1987: Erfolgswege zum neuen Arbeitsplatz. Wie Beschäftigte, die den Arbeitgeber wechselten, ihre neue Stelle fanden. Mitteilungen aus der Arbeitsmarkt- und Berufsforschung 20: $164-180$.

Brandt, Martina, 2004: Ausstieg aus Erwerbslosigkeit mithilfe sozialer Beziehungen. Eine Analyse mit den Daten des Niedrigeinkommens-Panels. Unveröffentlichte Magisterarbeit. Universität zu Köln: Lehrstuhl für empirische Sozial- und Wirtschaftsforschung.

Burt, Ronald S., 1983: Range. S. 176-194 in: Ronald S. Burt und Michael J. Minor (Hg.), Applied Network Analysis. A Methodological Introduction. Beverly Hills/London: Sage Publications.

Burt, Ronald S., 1992: Structural Holes. The Social Structure of Competition. Harvard: Harvard University Press.

Deeke, Axel, 1991: Informelle Beziehungen auf dem Arbeitsmarkt: Marktregulierung und Chancenverteilung durch Arbeitsvermittlung. Frankfurt a.M./New York: Campus Verlag. 
De Graaf, Nan D., und Hendrik D. Flap, 1988: „With a Little Help from my Friends“: Social Resources as an Explanation of Occupational Status and Income in West Germany, the Netherlands, and the United States. Social Forces 67, 1: 452-472.

Diewald, Martin, 1991: Soziale Beziehungen: Verlust oder Liberalisierung? Soziale Unterstützung in informellen Netzwerken. Berlin: Edition Sigma.

Friedkin, Noah E., 1982: Information Flow through Strong and Weak Ties in Intraorganizational Social Networks. Social Networks 3: 273-285.

Gilberg, Reiner, Doris Hess und Helmut Schröder, 2001: Struktur der Arbeitslosigkeit im Frühjahr 2000. Suchverhalten und Eingliederungschancen. Mitteilungen aus der Arbeitsmarkt- und Berufsforschung 34: 376-401.

Granovetter, Mark S., 1973: The Strength of Weak Ties. American Journal of Sociology 78, 6: $1360-1380$.

Granovetter, Mark S., 1974: Getting a Job: A Study of Contacts and Careers. Cambridge, MA: Harvard University Press.

Granovetter, Mark S., 1983: The Strength of Weak Ties. A Network Theory Revisited. S. 201-233 in: Randall Collins (Hg.), Sociological Theory 1983, 1. San Francisco: Jossey-Bass.

Granovetter, Mark S., 1992: Economic Action and Social Structure. The Problem of Embeddedness. S. 53-81 in: Mark S. Granovetter und Richard Swedberg (Hg.), The Sociology of Economic Life. Colorado: Westview Press.

Granovetter, Mark S., 1995: Getting a Job. A Study of Contacts and Careers. 2. Aufl. Chicago/London: University of Chicago Press.

Granovetter, Mark S., und Richard Swedberg (Hg.), 1992: The Sociology of Economic Life. Colorado: Westview Press.

Gröhnke, Klaus, Hermann Strasser, Thomas Bongartz, Gabriele Klein und Thomas Schweer, 1996: Soziale Netzwerke bei Langzeitarbeitslosen. Duisburger Beiträge zur soziologischen Forschung 2. Gerhard-Mercator-Universität Duisburg.

Gröhnke, Klaus, und Hermann Strasser, 1997: Die Arbeitsplatzsuchstrategien von Arbeitslosen. Forschungsprojekt Langzeitarbeitslosigkeit. Duisburger Beiträge zur soziologischen Forschung 5. Gerhard-Mercator-Universität Duisburg.

Grund, Christian, 2001: Zum Signalcharakter unterschiedlicher Wege der Stellenfindung. Die Betriebswirtschaft 61: 519-525.

Habich, Roland, 1987: Wege der Stellenfindung und berufliche Platzierung. S. 143-173 in: Axel Deeke, Joachim Fischer und Ursula Schumm-Garling (Hg.), Arbeitsmarktbewegung als sozialer Prozess. SAMF-Arbeitspapier 3. Paderborn.

Haug, Sonja, und Per Kropp, 2002: Soziale Netzwerke und der Berufseinstieg von Akademikern. Eine Untersuchung ehemaliger Studierender an der Fakultät für Sozialwissenschaften und Philosophie in Leipzig. Arbeitsbericht des Instituts für Soziologie 32. Abgerufen am 02.12 .03 über: http://www.uni-leipzig.de/ -sozio/main/f-projekte.html.

Jansen, Dorothea, 2003: Einführung in die Netzwerkanalyse. 2. Aufl. Opladen: Leske + Budrich.

Kaufmann, Franz Xaver, 1995: Zukunft der Familie im vereinten Deutschland. Gesellschaftliche und politische Bedingungen. München: C. H. Beck.

Kortmann, Klaus, und Peter Sopp, 2000: Lebenslagen in Deutschland. Die Bevölkerung im unteren Einkommensbereich. Demographische Strukturen, Einstiegsgründe und Ausstiegsdynamik. Ergebnisse des Niedrigeinkommens-Panels (NIEP). Forschungsprojekt im Rahmen des Armutsund Reichtumsberichts 2001. Bonn: Bundesministerium für Arbeit und Sozialordnung.

Kortmann, Klaus, Peter P. Sopp und Miriam Thum, 2002: Das Niedrigeinkommens-Panel (NIEP). Methodenbericht. Gesamtuntersuchung. Eine Untersuchung im Auftrag des Bundesministeriums für Gesundheit und Soziale Sicherung durchgeführt von Infratest Sozialforschung. München.

Lin, Nan, 2001: Social Capital. A Theory of Social Structure and Action. Cambridge: Cambridge University Press.

Liu, William T., und Robert W. Duff, 1972: The Strength in Weak Ties. Public Opinion Quarterly 36: 361-366. 
Niejahr, Elisabeth, 2003: hartz-reform. Zu viel Arbeit fürs Amt. DIE ZEIT 21.08.03 Nr. 35. Abgerufen am 12.08.04 über: http://www.zeit.de/2003/35/Arbeitsmarkt.

Niejahr, Elisabeth, und Kolja Rudzio, 2005: Leben mit der Arbeitslosigkeit. Alle Parteien versprechen neue Jobs. Doch Millionen Menschen werden noch lange leer ausgehen. Was soll mit ihnen geschehen? DIE ZEIT 15.09.05 Nr. 38. Abgerufen am 30.03.06 über: http://www.zeit.de/2005/ 38/Arbeitslose.

Noll, Heinz-Herbert, 1985: Arbeitsplatzsuche und Stellenfindung. S. 275-303 in: Helmut Knepel und Reinhard Hujer (Hg.), Mobilitätsprozesse auf dem Arbeitsmarkt. Frankfurt a.M./New York: Campus.

Noll, Heinz-Herbert, und Stefan Weick, 2002: Informelle Kontakte für Zugang zu Jobs wichtiger als Arbeitsvermittlung. Prozesse der Arbeitssuche und Stellenfindung im europäischen Vergleich. Sozialberichterstattung, gesellschaftliche Trends, aktuelle Informationen. Informationsdienst Soziale Indikatoren ISI 28. Mannheim: ZUMA 6-10.

Pischner, Rainer, Jürgen Schupp und Gert G. Wagner, 2002: Arbeitsvermittlung durch das Arbeitsamt: Reform des Berichtssystems dringend erforderlich. DIW Wochenbericht 69, 9: 145-152.

Preisendörfer, Peter, und Thomas Voss, 1988: Arbeitsmarkt und soziale Netzwerke. Die Bedeutung sozialer Kontakte beim Zugang zu Arbeitsplätzen. Soziale Welt 39: 104-119.

Runia, Peter, 2002a: Das soziale Kapital auf dem Arbeitsmarkt: Beziehungen in Stellensuche, Personalrekrutierung und Beförderung. Europäische Hochschulschriften Soziologie, Bd. 366. Frankfurt a.M.: Peter Lang.

Runia, Peter, 2002b: Arbeitsmarkt und soziales Kapital. Eine komprimierte Darstellung theoretischer Grundlagen und empirischer Befunde. Duisburger Beiträge zur soziologischen Forschung 1. Gerhard-Mercator-Universität Duisburg.

Tazelaar, Frits, 1990: Die Verarbeitung langfristiger Arbeitslosigkeit. S. 191-228 in: Karl-Dieter Opp und Reinhard Wippler (Hg.), Empirischer Theorienvergleich. Erklärungen sozialen Verhaltens in Problemsituationen. Opladen: Westdeutscher Verlag.

Uhlendorff, Arne, 2004: Der Einfluss von Persönlichkeitseigenschaften und sozialen Ressourcen auf die Arbeitslosigkeitsdauer. Kölner Zeitschrift für Soziologie und Sozialpsychologie 56: 279-303.

Vishwanath, Tara, 1989: Job Search, Stigma Effect and Escape Rate from Unemployment. Journal of Labor Economics 7: 487-502.

Wegener, Bernd, 1987: Vom Nutzen entfernter Bekannter. Kölner Zeitschrift für Soziologie und Sozialpsychologie 39: 278-301.

Wegener, Bernd, 1989: Soziale Beziehungen im Karriereprozeß. Kölner Zeitschrift für Soziologie und Sozialpsychologie 41: 270-297.

Wegener, Bernd, 1991: Job Mobility and Social Ties: Social Resources, Prior Job, and Status Attainment. American Sociological Review 56: 60-71.

Windolf, Paul, und Hans-Willy Hohn, 1984: Arbeitsmarktchancen in der Krise. Betriebliche Rekrutierung und soziale Schließung. Frankfurt a.M./New York: Campus.

Windolf, Paul, und Hans-Willy Hohn, 1985: Prozesse sozialer Schließung im Arbeitsmarkt. S. 305327 in: Helmut Knepel und Reinhard Hujer (Hg.), Mobilitätsprozesse auf dem Arbeitsmarkt. Frankfurt a.M./New York: Campus.

Wooldridge, Jeffrey, 2003: Introductory Econometrics: A Modern Approach. South Western College Publishing.

Yamaguchi, Kazuo, 1991: Event History Analysis. Applied Social Research Methods Series 28. Newbury Park/London: Sage.

Korrespondenzanschrift: Martina Brandt, Universität Zürich, Soziologisches Institut, Andreasstr. 15, CH-8050 Zürich

E-Mail: brandt@soziologie.unizh.ch 